\title{
Effective string theory simplified
}

\section{Simeon Hellerman, ${ }^{a}$ Shunsuke Maeda, ${ }^{a, b}$ Jonathan Maltz ${ }^{a}$ and Ian Swanson}

${ }^{a}$ Kavli Institute for the Physics and Mathematics of the Universe, The University of Tokyo, Kashiwa, Chiba 277-8582, Japan

${ }^{b}$ Department of Physics, Faculty of Science, University of Tokyo, Bunkyo-ku, Tokyo 133-0022, Japan

E-mail: simeon.hellerman.1@gmail.com, shunsuke.maeda@ipmu.jp, jdmaltz@alumni.stanford.edu, ianswanson.physics@gmail.com

ABstract: In this note we simplify the formulation of the Poincaré-invariant effective string theory in $D$ dimensions by adding an intrinsic metric and embedding its dynamics into the Polyakov formalism. We use this formalism to classify operators order-by-order in the inverse physical length of the string, in a fully gauge-invariant framework. We then use this classification to analyze the universality and nonuniversality of observables, up to and including the second sub-leading order in the long string expansion.

KEYWORDS: Bosonic Strings, Long strings

ARXIV EPRINT: 1405.6197 


\section{Contents}

1 Introduction 1

2 Effective string theory in the Polyakov formalism 2

2.1 Generalities 2

2.2 Stress tensor in the effective string in Polyakov formalism 4

$\begin{array}{lll}2.3 & \text { EOM and stress tensor conservation } & 7\end{array}$

2.3.1 Deriving the EOM 7

2.3.2 Verifying the holomorphy of the stress tensor 8

2.4 Relation to the old covariant formalism 8

3 Local operators in covariant effective string theory 9

$\begin{array}{lll}3.1 & \text { Weyl tensor calculus on the worldsheet } & 9\end{array}$

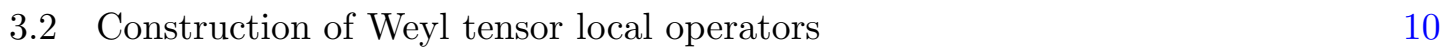

3.3 Higher-dimension operators in the string effective action 11

$\begin{array}{lll}\text { 3.3.1 Conformal dimension and } X \text {-scaling } & 11\end{array}$

$\begin{array}{lll}\text { 3.3.2 The } \mathcal{I}_{11} \text {-dressing rule } & 12\end{array}$

$\begin{array}{lll}\text { 3.3.3 Scalar operators of conformal dimension } 2 & 13\end{array}$

$\begin{array}{lll}3.4 & \text { Universality and nonuniversality } & 14\end{array}$

$\begin{array}{lll}3.4 .1 & \text { Universality at NLO } & 14\end{array}$

$\begin{array}{lll}3.4 .2 & \text { Nonuniversality at NNLO } & 15\end{array}$

4 Interaction corrections to the OPE $\quad 16$

$\begin{array}{lll}4.1 & \text { The propagator correction } & 16\end{array}$

4.2 Nonrenormalization of the OPE between $T$ and $X$ at $O\left(\beta^{1}\right) \quad 16$

$\begin{array}{lll}4.3 & \text { Closure of the OPE of } T \text { with itself } & 18\end{array}$

$\begin{array}{lll}\text { 4.4 OPE of } T \text { with composite operators } & 18\end{array}$

$\begin{array}{llr}5 & \text { Conclusions } & 19\end{array}$

\section{Introduction}

String theory was originally developed as a tool to study the dynamics of chromoelectric flux tubes in strongly coupled gauge theory, but it quickly got sort of sidetracked into the study of gravity. Some time later, a consistent quantization was developed [1] for Poincaréinvariant string theories in $D \neq 26$, in the limit where the physical scale of the string is much larger than the square root of the inverse string tension, or $\sqrt{\alpha^{\prime}}$. Decades after the original discovery of string theory, the study of gravity led back to the application of string theory to strongly coupled gauge theory, via gauge-gravity duality and the holographic principle [2]. 
The relationship between holographic string duals of confining gauge theories and effective non-gravitational string theory was to some extent worked out in [3], drawing on earlier work on the derivation of effective string theories from fundamental strings propagating on warped geometries [4] and perturbed Liouville theories [1].

The effective theory of relativistic strings has developed in spite of the lack of a truly simple formalism. Static gauge [5-7] lacks manifest covariance, and calculations in static gauge at the quantum level are vulnerable to subtle errors due to Lorentz-breaking effects entering through the procedures used to regularize and renormalize the theory. The Polchinski-Strominger (PS) formalism, while manifestly relativistically covariant and relatively easy to quantize, is based on the ad hoc addition of a singular interaction to the gauge-fixed Lagrangian.

Therefore, it is desirable to develop a more systematic treatment of the quantization of string dynamics in the effective framework prior to gauge fixing, so that the translation between different gauges and renormalization schemes can be carried out with ease and clarity. In this paper we develop such an approach, by embedding the effective string into the Polyakov formalism. (We also direct the reader's attention to the earlier work [8], where an embedding of the effective string into the Polyakov path integral was also pursued. We thank N. D. Hari Dass for making us aware of this work.)

\section{Effective string theory in the Polyakov formalism}

\subsection{Generalities}

The Polyakov string is defined by the path integral

$$
\begin{aligned}
Z & =\int \mathcal{D} \mu_{[g]}^{\text {Polyakov }} \exp \left\{-S_{\text {Polyakov }}\right\}, \\
\mathcal{D} \mu_{[g]}^{\text {Polyakov }} & \equiv \frac{\mathcal{D}_{[g]} X \mathcal{D}_{[g]} g}{\mathcal{D}_{[g]} \Omega}, \\
S_{\text {Polyakov }} & =\int d^{2} \sigma \sqrt{\left|g_{\bullet \bullet}\right|} \mathcal{L}_{\text {Polyakov }}, \\
\mathcal{L}_{\text {Polyakov }} & =\frac{1}{4 \pi \alpha^{\prime}} g^{a b} \partial_{a} X^{\mu} \partial_{b} X_{\mu} .
\end{aligned}
$$

The subscript $[g]$ on the various factors of the path integral measure mean that the various pieces of the measure are regularized and renormalized with a local prescription using the fiducial metric $g_{\bullet \bullet}$.

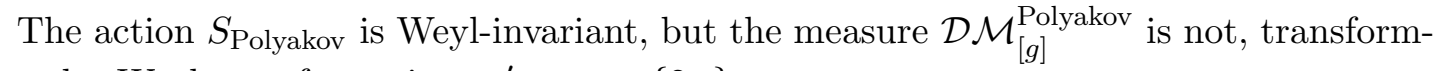
ing under Weyl transformations $g_{\bullet \bullet}^{\prime}=\exp \{2 \omega\} g_{\bullet \bullet}$ as

$$
\mathcal{D} \mathcal{M}_{\left[g^{\prime}\right]}^{\text {Polyakov }}=\exp \left\{\frac{D-26}{24 \pi} \int d^{2} \sigma \sqrt{|g|}\left(g^{a b} \partial_{a} \omega \partial_{b} \omega+\omega \mathcal{R}_{(2)}\right)\right\} \cdot \mathcal{D} \mathcal{M}_{[g]}^{\text {Polyakov }},
$$

in a general number of dimensions $D$. The expression above is based on a Euclideansignature worldsheet. For a Lorentzian-signature worldsheet metric $g$ (with $g_{00}<0$ signature convention), we have

$$
\mathcal{D} \mathcal{M}_{\left[g^{\prime}\right]}^{\text {Polyakov }}=\exp \left\{i \frac{D-26}{24 \pi} \int d \sigma^{0} d \sigma^{1} \sqrt{|g|}\left(g^{a b} \partial_{a} \omega \partial_{b} \omega+\omega \mathcal{R}_{(2)}\right)\right\} \cdot \mathcal{D} \mathcal{M}_{[g]}^{\text {Polyakov }} .
$$


In the "linear dilaton" or "quantum Liouville theory" approach, one cancels this anomaly by assigning a nontrivial Weyl transformation to one of the scalars $X^{D-1} \equiv$ $\frac{1}{|V|} V_{\mu} X^{\mu}$ :

$$
X^{D-1} \rightarrow X^{D-1}-\sqrt{\frac{26-D}{6 \alpha^{\prime}}} \omega .
$$

Then $X$ can be related to the Liouville field $\phi$, which transforms under Weyl transformations with a unit shift, by

$$
X^{D-1}=-\sqrt{\frac{26-D}{6 \alpha^{\prime}}} \phi, \quad \phi=-\sqrt{\frac{6 \alpha^{\prime}}{26-D}} X^{D-1} .
$$

The transformation of $\phi$ is thus

$$
\phi \rightarrow \phi+\omega
$$

and the action for $\phi$ (in Euclidean signature) is

$$
S_{\phi}=\frac{26-D}{24 \pi} \int d^{2} \sigma \sqrt{|g|}\left(g^{a b} \partial_{a} \phi \partial_{b} \phi-\phi \mathcal{R}_{(2)}\right),
$$

or

$$
S_{\phi}=\frac{D-26}{24 \pi} \int d \sigma^{0} d \sigma^{1} \sqrt{|g|}\left(g^{a b} \partial_{a} \phi \partial_{b} \phi-\phi \mathcal{R}_{(2)}\right)
$$

in Lorentzian signature $\left(g_{00}<0\right)$. The anomalous transformation of the measure is then cancelled exactly by the classical transformation of the action for $\phi$. The latter depends only on the transformation of the $\phi$ field itself. Therefore, we can achieve the exact same classical transformation of the action by substituting any composite operator $\varphi$ for $\phi$ that transforms as

$$
\varphi \rightarrow \varphi+\omega
$$

under a Weyl transformation, and transforms as a scalar under diffeomorphisms. One can easily construct such operators from $X^{\mu}$ and $g_{a b}$, while leaving all $X^{\mu}$ to transform trivially under Weyl transformations, thus preserving $D$-dimensional Poincaré invariance. The simplest scalar operator that transforms as (2.3), is

$$
\varphi \equiv-\frac{1}{2} \ln \left(g^{a b} \partial_{a} X^{\mu} \partial_{b} X_{\mu}\right) .
$$

So, in terms of $\varphi$, our action becomes

$$
S=S_{\text {Polyakov }}+S_{\substack{\text { composite } \\ \text { Liouville }}}
$$

where

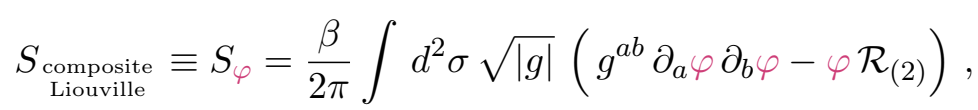

where we have defined the coefficient

$$
\beta \equiv \frac{26-D}{12} .
$$


We have written the coefficient of the anomaly action in terms of $\beta$ to connect later on with the conventions and notation [1] of the old covariant formalism. ${ }^{1}$

As noted, with this definition of the action, the anomalous transformation (2.1) of the measure $\mathcal{D} \mathcal{M}_{[g]}^{\text {Polyakov }}$ is precisely cancelled by the classical transformation of the action (2.5), and the path integral is invariant. Of course there are many (indeed, an infinite number of) additional diff $\times$ Weyl-invariant terms one could add to the action, and, correspondingly, many (an infinite number of) ambiguities in the definition of the theory arising from dependence on the regularization and renormalization scheme. These two sets of ambiguities are of course the same: any two local regularization and renormalization procedures must differ in effect by local terms in the bare action. Any two renormalization schemes that properly preserve all the symmetries, including diff $\times$ Weyl invariance, must differ by local terms that preserve the same symmetries. However, there is no local term preserving all symmetries that scales as $|X|^{0}$ in the long string expansion, other than the Euler density, which is topological. Thus, any two consistent renormalization schemes must yield equivalent amplitudes for all processes at first subleading order in the long-string expansion, modulo a possible renormalization of the effective string coupling for processes involving scattering or decays. In particular, there can be no disagreement among observable processes for different "quantizations" of the effective string at first subleading order, so long as the symmetries are properly preserved. ${ }^{2}$

\subsection{Stress tensor in the effective string in Polyakov formalism}

The major difference between the linear dilaton case and the composite-Liouville case is that the composite Liouville field $\varphi$ itself involves the metric in its definition. That is, suppose we vary the metric by an infinitesimal amount

$$
g^{a b} \rightarrow g^{a b}+h^{a b}, \quad g_{a b} \rightarrow g_{a b}-h_{a b}, \quad h_{a b} \equiv g_{a c} g_{b d} h^{c d},
$$

whereby $\frac{\delta}{\delta h^{a b}} \varphi \neq 0$. Note that this is in contrast to the case of a conventional Liouville field $\phi$, which is an independent degree of freedom defined without respect to the metric. In particular, with the definition (2.4), we have

$$
\frac{\delta \varphi}{\delta h^{a b}}=-\frac{1}{2} \frac{\partial_{a} X \cdot \partial_{b} X}{(\nabla X)^{2}}
$$

We can thus write expressions for the variation of the kinetic term and the Ricci coupling for the composite Liouville field $\varphi$. To this end, we apply standard formulae from differential geometry, remembering to supplement terms from the explicit metric variation of the action at fixed $\varphi$ with terms coming from the metric variation of $\varphi$ itself, shown in eq. (2.8).

Defining

$$
S^{(\mathrm{kin})} \equiv \frac{26-D}{24 \pi} \int \sqrt{|g|} d^{2} \sigma g^{a b} \partial_{a} \varphi \partial_{b} \varphi,
$$

\footnotetext{
${ }^{1}$ Note that there is a sign error in the equation expressing the value of $\beta$ in [1]. The sign of $\beta$ in our (2.7) is the correct one, and is in agreement with all calculations and equations of [1], other than their equation (15). The incorrect sign in that expression appears to be a simple typographical error.

${ }^{2}$ For a beautiful analysis of matching between various gauges and renormalization schemes, the interested reader is urged to consult $[3,9]$.
} 
and

$$
S^{(\text {Ricci })} \equiv-\frac{26-D}{24 \pi} \int d^{2} \sigma \sqrt{|g|} \varphi \mathcal{R}_{(2)}
$$

we find the contributions to the stress tensor at order $\beta^{1}|X|^{0}$ by taking the variation of the order- $\beta^{1}|X|^{0}$ piece of the worldsheet action, which is just the anomaly-cancelling term $S^{(\text {kin })}+S^{(\text {Ricci) }}$. Defining $T_{a b}^{\left[\beta^{1}|X|^{0}\right]} \equiv-4 \pi \frac{\delta}{\delta h^{a b}} S_{\substack{\text { composite } \\ \text { Liouvile }}}$ and using (2.8), we find

$$
T_{a b(\varphi)}^{\left[\beta^{1}|X|^{0}\right]}=T_{a b(\varphi)}^{(\text {kin })}+T_{a b(\varphi)}^{(\text {Ricci) }}
$$

where

$$
\begin{aligned}
& T_{a b(\varphi)}^{(\mathrm{kin})}=\frac{26-D}{6}\left[-\left(\nabla_{a} \varphi \nabla_{b} \varphi-\frac{1}{2} g_{a b}(\nabla \varphi)^{2}\right)-\left(\nabla^{2} \varphi\right)\left(+\frac{\nabla_{a} X \cdot \nabla_{b} X}{(\nabla X)^{2}}\right)\right], \\
& T_{a b(\varphi)}^{(\mathrm{Ricci})}=\frac{D-26}{6}\left[\nabla_{a} \nabla_{b} \varphi-g_{a b} \nabla^{c} \nabla_{c} \varphi+\frac{1}{2} \frac{\partial_{a} X \cdot \partial_{b} X}{(\nabla X)^{2}} \mathcal{R}_{(2)}\right] .
\end{aligned}
$$

The trace of the two pieces above appear (classically) as

$$
\begin{aligned}
T_{(\varphi)}^{(\mathrm{kin}){ }_{a}{ }_{a}} & =g^{a b} T_{a b(\varphi)}^{(\mathrm{kin})}=-\frac{26-D}{6} \nabla^{2} \varphi \\
T_{(\varphi)}^{(\mathrm{Ricci}) a}{ }_{a} & =g^{a b} T_{a b(\varphi)}^{(\mathrm{Ricci})}=+\frac{26-D}{6} \nabla^{2} \varphi-\frac{26-D}{12} \mathcal{R}_{(2)} .
\end{aligned}
$$

So the trace of the total order- $\beta^{1}|X|^{0}$ stress tensor (classically) is

$$
T_{(\varphi)}^{\left[\beta^{1}|X|^{0}\right]_{a}}{ }_{a}=-\frac{26-D}{12} \mathcal{R}_{(2)}, \quad \text { (modulo quantum corrections) }
$$

The quantum correction, given by equation (3.4.15) of [22], is

$$
T_{a}^{(\text {quantum }) a}=-\frac{c}{12} \mathcal{R}_{(2)},
$$

where, for us, $c=D-26$. We then have

$$
T_{a}^{(\text {quantum }) a}=\frac{26-D}{12} \mathcal{R}_{(2)} .
$$

Thus, the total (classical plus quantum) trace of the stress tensor is

$$
\left.T_{a}^{a}\right|_{(\varphi)} ^{\left(\text {classical, } O\left(\beta^{1}|X|^{0}\right)\right)}+\left.T_{a}^{a}\right|^{(\text {quantum })}=0 .
$$

For a flat metric in Euclidean signature, $g_{a b}=\delta_{a b}$, meaning $\delta_{w w}=\delta_{\bar{w} \bar{w}}=0$ and $\delta_{w \bar{w}}=\delta_{\bar{w} w}=\frac{1}{2}$ in the standard complex coordinates

$$
\begin{aligned}
& w \equiv \sigma^{2}+i \sigma^{1}, \\
& \bar{w} \equiv \sigma^{2}-i \sigma^{1} .
\end{aligned}
$$

\footnotetext{
${ }^{3}$ In accordance with the sign and normalization conventions of [1] for the stress tensor.
} 
Then $\mathcal{R}_{(2)}=0$ and we have

$$
\begin{aligned}
T_{w w(\varphi)}^{(\mathrm{kin})} & =\frac{26-D}{6}\left[-\partial_{w} \varphi \partial_{w} \varphi-\left(\nabla^{2} \varphi\right)\left(\frac{\partial_{w} X \cdot \partial_{w} X}{(\nabla X)^{2}}\right)\right], \\
T_{w w(\varphi)}^{(\mathrm{Ricci})} & =-\frac{26-D}{6} \partial_{w}^{2} \varphi .
\end{aligned}
$$

Then, using

$$
\nabla^{2}=4 \partial_{w} \partial_{\bar{w}}
$$

and

$$
\nabla X \cdot \nabla X=4 \partial_{w} X \cdot \partial_{\bar{w}} X
$$

we have

$$
\begin{aligned}
T_{w w(\varphi)}^{(\mathrm{kin})} & =\frac{26-D}{6}\left[-\partial_{w} \varphi \partial_{w} \varphi+\left(\partial_{w} \partial_{\bar{w}} \varphi\right)\left(\frac{\partial_{w} X \cdot \partial_{w} X}{\partial_{w} X \cdot \partial_{\bar{w}} X}\right)\right], \\
T_{w w(\varphi)}^{(\mathrm{Ricci})} & =-\frac{26-D}{6} \partial_{w}^{2} \varphi .
\end{aligned}
$$

For brevity, we write $\partial_{w} \equiv \partial$ and $\partial_{\bar{w}} \equiv \bar{\partial}$, so that

$$
\begin{aligned}
T_{w w(\varphi)}^{(\text {kin })} & =\frac{26-D}{6}\left[-(\partial \varphi)^{2}+(\partial \bar{\partial} \varphi)\left(\frac{(\partial X)^{2}}{\partial X \cdot \bar{\partial} X}\right)\right], \\
T_{w w(\varphi)}^{(\text {Ricci })} & =-\frac{26-D}{6} \partial^{2} \varphi .
\end{aligned}
$$

For $g_{a b}=\delta_{a b}$ we have $\varphi=-\frac{1}{2} \ln (4 \partial X \cdot \bar{\partial} X)=-\frac{1}{2} \ln (\partial X \cdot \bar{\partial} X)+$ const.. So, discarding terms proportional to the leading order equations of motion (that is, setting $\partial \bar{\partial} X=O\left(\beta X^{-1}\right)$ by the EOM), we have

$$
\begin{aligned}
\partial \varphi & =-\frac{1}{2} \frac{\partial^{2} X \cdot \bar{\partial} X}{\partial X \cdot \bar{\partial} X} \\
\partial^{2} \varphi & =-\frac{1}{2} \frac{\partial^{3} X \cdot \bar{\partial} X}{\partial X \cdot \bar{\partial} X}+\frac{1}{2} \frac{\left(\partial^{2} X \cdot \bar{\partial} X\right)^{2}}{(\partial X \cdot \bar{\partial} X)^{2}}
\end{aligned}
$$

and

$$
\partial \bar{\partial} \varphi=-\frac{1}{2} \frac{\partial^{2} X \cdot \bar{\partial}^{2} X}{\partial X \cdot \bar{\partial} X}+\frac{1}{2} \frac{\left(\partial^{2} X \cdot \bar{\partial} X\right)\left(\partial X \cdot \bar{\partial}^{2} X\right)}{(\partial X \cdot \bar{\partial} X)^{2}} .
$$

Using the notation $\mathcal{I}_{p q} \equiv \partial^{p} X \cdot \bar{\partial}^{q} X$ in Euclidean signature (or $\mathcal{I}_{p q} \equiv \partial_{+}^{p} X \cdot \partial_{-}^{q} X$ for Lorentzian signature), we have

$$
\begin{aligned}
\partial \varphi & =-\frac{1}{2} \frac{\mathcal{I}_{21}}{\mathcal{I}_{11}}, \\
\partial^{2} \varphi & =-\frac{1}{2} \frac{\mathcal{I}_{31}}{\mathcal{I}_{11}}+\frac{1}{2} \frac{\mathcal{I}_{21}^{2}}{\mathcal{I}_{11}^{2}},
\end{aligned}
$$

and

such that

$$
\partial \bar{\partial} \varphi=-\frac{1}{2} \frac{\mathcal{I}_{22}}{\mathcal{I}_{11}}+\frac{1}{2} \frac{\mathcal{I}_{21} \mathcal{I}_{12}}{\mathcal{I}_{11}^{2}}
$$

$$
(\partial \bar{\partial} \varphi)\left(\frac{(\partial X)^{2}}{\partial X \cdot \bar{\partial} X}\right)=(\partial X)^{2}\left(-\frac{1}{2} \frac{\mathcal{I}_{22}}{\mathcal{I}_{11}^{2}}+\frac{1}{2} \frac{\mathcal{I}_{21} \mathcal{I}_{12}}{\mathcal{I}_{11}^{3}}\right)
$$




\subsection{EOM and stress tensor conservation}

Next we will write the EOM for the $X^{\mu}$ coordinates and verify the classical conservation of the stress tensor, when the EOM is satisfied.

\subsubsection{Deriving the EOM}

Under a general variation of $X, X^{\mu} \rightarrow X^{\mu}+\epsilon^{\mu}$, the field $\varphi$ varies as

$$
\delta \varphi=-\frac{\nabla X \cdot \nabla \epsilon}{(\nabla X)^{2}}
$$

The variation of the action is

$$
\begin{aligned}
0=-\pi \alpha^{\prime} \delta S_{\mathrm{E}}= & \int d^{2} \sigma \sqrt{|g|} \epsilon_{\mu}\left\{\frac{1}{2} \nabla^{2} X^{\mu}+\beta \alpha^{\prime} \nabla^{a}\left[\frac{\nabla_{a} X^{\mu}}{(\nabla X)^{2}} \nabla^{2} \varphi\right]\right\} \\
& +\left(\text { terms involving } \mathcal{R}_{(2)}\right),
\end{aligned}
$$

so the EOM is

$$
\nabla^{2} X^{\mu}=-2 \beta \alpha^{\prime} \nabla^{a}\left[\frac{\nabla_{a} X^{\mu}}{(\nabla X)^{2}} \nabla^{2} \varphi\right]+\left(\text { terms involving } \mathcal{R}_{(2)}\right) .
$$

On flat space we have

$$
\vec{\partial}^{2} X^{\mu}=-2 \beta \alpha^{\prime} \partial^{a}\left[\frac{\partial_{a} X^{\mu}}{(\vec{\partial} X)^{2}} \vec{\partial}^{2} \varphi\right]
$$

where $\vec{\partial}^{2}$ is the flat Laplacian $\partial_{b} \partial_{b}$. Written in terms of $w$ and $\bar{w}$, we have

$$
\partial \bar{\partial} X^{\mu}=-\beta \alpha^{\prime} \partial\left[\frac{\bar{\partial} X^{\mu}}{\mathcal{I}_{11}} \partial \bar{\partial} \varphi\right]-\beta \alpha^{\prime} \bar{\partial}\left[\frac{\partial X^{\mu}}{\mathcal{I}_{11}} \partial \bar{\partial} \varphi\right] .
$$

It is helpful to recall the usual logic about why we can discard terms proportional to $\partial \bar{\partial} X$ on the right-hand side, if we are only working to order $\beta|X|^{-2}$ relative to leadingorder quantities. The logic is that our EOM are of the form $\partial \bar{\partial} X^{\mu}=O(\beta /|X|)$. Now the order $\beta /|X|$ terms on the right-hand side can be separated into terms without $\partial \bar{\partial} X$ factors, and terms with $\partial \bar{\partial} X$ factors. Both are at most of order $\beta /|X|$, of course, but the latter is equal to $(\partial \bar{\partial} X)^{\nu} S_{\nu}$, where $S_{\nu}$ is of order $\beta /|X|^{2}$. However, $\partial \bar{\partial} X$ is itself of order $\beta /|X|$. We then have

$\partial \bar{\partial} X^{\mu}=\{$ terms obtained by discarding $\partial \bar{\partial} X$ on the r.h.s. of $(2.14)\}+O\left(\beta^{2} /|X|^{3}\right)$.

Letting the equivalence $\simeq$ denote equality up to terms of $O\left(\beta^{2} /|X|^{3}\right)$, we obtain

$$
\partial \bar{\partial} X^{\mu} \simeq-\beta \alpha^{\prime}\left(\bar{\partial} X^{\mu} \partial\left[\frac{1}{\mathcal{I}_{11}} \partial \bar{\partial} \varphi\right]+\partial X^{\mu} \bar{\partial}\left[\frac{1}{\mathcal{I}_{11}} \partial \bar{\partial} \varphi\right]\right)
$$




\subsubsection{Verifying the holomorphy of the stress tensor}

To evaluate the nonconservation of the order- $\beta^{0}$ holomorphic stress tensor, we can contract (2.15) with $-\frac{2}{\alpha^{\prime}} \partial X_{\mu}$, to obtain:

$$
\begin{aligned}
\bar{\partial} T^{\left[\beta^{0}\right]} & =-\frac{1}{\alpha^{\prime}} \bar{\partial}(\partial X \cdot \partial X)=-\frac{2}{\alpha^{\prime}} \partial X_{\mu} \cdot \partial \bar{\partial} X^{\mu} \\
& \simeq 2 \beta \partial X_{\mu}\left\{\bar{\partial}\left[\frac{\partial \bar{\partial} \varphi}{\mathcal{I}_{11}} \partial X^{\mu}\right]+\partial\left[\frac{\partial \bar{\partial} \varphi}{\mathcal{I}_{11}} \bar{\partial} X^{\mu}\right]\right\} \\
& =2 \beta\left\{\frac{\bar{\partial}(\partial X \cdot \partial X)}{\mathcal{I}_{11}} \partial \bar{\partial} \varphi+\partial^{2} \bar{\partial} \varphi-\frac{\partial \mathcal{I}_{11}}{\mathcal{I}_{11}} \partial \bar{\partial} \varphi+\frac{\partial X \cdot \partial X}{\mathcal{I}_{11}} \partial \bar{\partial}^{2} \varphi-\frac{\partial X \cdot \partial X}{\mathcal{I}_{11}{ }^{2}} \bar{\partial} \mathcal{I}_{11} \partial \bar{\partial} \varphi\right\} .
\end{aligned}
$$

Now we can also evaluate the nonconservation of the order- $\beta^{1}$ piece of the stress tensor. Using $\frac{26-D}{6}=+2 \beta$, we have

$$
\begin{aligned}
\bar{\partial} T^{\left[\beta^{1}\right]} & =-2 \beta \bar{\partial}\left\{(\partial \varphi)^{2}+\frac{(\partial X)^{2} \partial \bar{\partial} \varphi}{\mathcal{I}_{11}}+\partial^{2} \varphi\right\} \\
& =-2 \beta\left\{2 \partial \varphi \partial \bar{\partial} \varphi+\frac{\bar{\partial}(\partial X \cdot \partial X)}{\mathcal{I}_{11}} \partial \bar{\partial} \varphi+\frac{\partial X \cdot \partial X}{\mathcal{I}_{11}} \partial \bar{\partial}^{2} \varphi-\partial X \cdot \partial X \partial \bar{\partial} \varphi \frac{\bar{\partial} \mathcal{I}_{11}}{\mathcal{I}_{11}{ }^{2}}+\partial^{2} \bar{\partial} \varphi\right\} .
\end{aligned}
$$

These two nonconservations cancel each other out exactly, so we conclude:

$$
\bar{\partial} T^{\left[\beta^{0}\right]}+\bar{\partial} T^{\left[\beta^{1}\right]} \simeq 0
$$

where the $\simeq$ denotes equality up to terms of order $\beta^{2} /|X|^{2}$.

\subsection{Relation to the old covariant formalism}

We now relate our embedding of the effective string in the Polyakov formalism to the old Lorentz-covariant formalism of [1]. Apart from the addition of the intrinsic metric and the corresponding Weyl invariance, the relationship between the two actions also includes a redefinition of the $X$ variables at next-to-leading order.

Our stress tensor is not conserved if we apply the EOM derived from the PolchinskiStrominger action in the form written in [1]. This has to do with the fact that our composite-Liouville action differs from the PS action by linear combinations of $\partial \bar{\partial} X^{\mu}$ multiplied by operators, meaning that the $X$-variables here differ from those in [1] by a field transformation of the form $X^{\mu} \rightarrow X^{\mu}+\mathcal{O}^{\mu}$, where

$$
\mathcal{O}^{\mu}=\frac{\beta \alpha^{\prime}}{4} \frac{1}{\mathcal{I}_{11}^{2}}\left\{\left(\mathcal{I}_{21}+\partial X \cdot \partial \bar{\partial} X\right) \bar{\partial} X^{\mu}+\mathcal{I}_{12} \partial X^{\mu}\right\} .
$$

To derive this, we first find terms of the form $F[X]^{\mu} \partial \bar{\partial} X_{\mu}$ in our composite-Liouville action. Then, $F[X]^{\mu}$ and $\mathcal{O}^{\mu}$ are related by $\mathcal{O}^{\mu}=-\pi \alpha^{\prime} F[X]^{\mu}$.

The useful aspect of this change of variables has to do with the conformal properties of the $X^{\mu}$ field. The worldsheet coordinate $X_{\mathrm{PS}}^{\mu}$ as defined in [1] does not have simple conformal properties. The operator product expansion between the stress tensor and $X_{\mathrm{PS}}^{\mu}$ has a singular term $z^{-3} \beta \frac{\bar{\partial} X^{\mu}}{\mathcal{I}_{11}}$, so the coordinate $X_{\mathrm{PS}}^{\mu}$ is not a conformal primary. We shall see in section (4.2) that our embedding coordinate $X_{\text {here }}^{\mu}$ has leading term $z^{-1} \partial X_{\text {here }}^{\mu}$ 
in its OPE with the stress tensor. The $X_{\text {here }}$ variables in our formalism are more natural objects from a conformal point of view, which follows from our derivation of the compositeLiouville interaction term from a Weyl-invariant path integral in which $X_{\text {here }}^{\mu}$ is taken to be invariant under Weyl rescalings. ${ }^{4}$ We shall establish the quantum conformal properties of our embedding coordinates $X_{\text {here }}^{\mu}$ by explicit calculation in section 4 .

\section{Local operators in covariant effective string theory}

Let us now classify Weyl-invariant operators, organizing them by their $X$-scaling. We begin by constructing a Weyl tensor calculus based on a Weyl-covariant derivative using the composite Liouville field $\varphi$. (Essentially the same Weyl tensor calculus was developed in [8].)

\subsection{Weyl tensor calculus on the worldsheet}

The ordinary Riemannian covariant derivative does not transform covariantly under Weyl transformations. The Riemannian connection transforms as

$$
\delta\left(\nabla_{b} V^{a}\right)=\left(\partial_{b} \omega\right) V^{a}+\delta^{a}{ }_{b}\left(V^{c} \partial_{c} \omega\right)-\left(\partial^{a} \omega\right) V_{b}
$$

under a Weyl transformation parametrized by $\omega$, if $V$ itself is neutral under Weyl rescalings. However, using the effective Liouville field $\varphi$, which simply shifts under a Weyl transformation (2.3), we can easily render our Riemannian connection Weyl-covariant. Defining the Weyl-covariant derivative

$$
\hat{\nabla}_{b} V^{a} \equiv \nabla_{b} V^{a}-\left(\partial_{b} \varphi\right) V^{a}-\delta^{a}{ }_{b}\left(V^{c} \partial_{c} \varphi\right)+\left(\partial^{a} \varphi\right) V_{b},
$$

$\hat{\nabla}_{b} V^{a}$ transforms as a tensor of type $(1,1)$ under diffeomorphisms, and trivially under Weyl transformations:

$$
\delta_{(\text {Weyl })} \hat{\nabla}_{b} V^{a}=0,
$$

if we assume the property (2.3). The action of our diff- and Weyl-covariant derivative on covectors is

$$
\hat{\nabla}_{a} W_{b} \equiv \nabla_{a} W_{b}+\left(\partial_{b} \varphi\right) W_{a}+\left(\partial_{a} \varphi\right) W_{b}-g_{a b}\left(W_{c} \partial^{c} \varphi\right),
$$

if $W$ transforms trivially under Weyl transformations. So, in particular, if $W_{b} \equiv \hat{\nabla}_{b} Y=$ $\nabla_{b} Y=\partial_{b} Y$ for some scalar $Y$, then

$$
\hat{\nabla}_{a} \hat{\nabla}_{b} Y=\hat{\nabla}_{a} \nabla_{b} Y=\nabla_{a} \nabla_{b} Y+\partial_{a} \varphi \partial_{b} Y+\partial_{b} \varphi \partial_{a} Y-g_{a b} \partial^{c} \varphi \partial_{c} Y .
$$

We conclude by commenting on this Weyl-covariant double derivative. First, it is automatically symmetric when acting on scalars, just like the Riemannian covariant derivative:

$$
\hat{\nabla}_{a} \hat{\nabla}_{b} Y=\hat{\nabla}_{b} \hat{\nabla}_{a} Y \text {. }
$$

Second, the correction terms implementing the Weyl-covariance are traceless, so the Weylcovariant Laplacian is equal to the ordinary Riemannian Laplacian:

$$
\hat{\nabla}^{a} \hat{\nabla}_{a} Y=\nabla^{a} \nabla_{a} Y \text {. }
$$

These identities will prove themselves convenient in the calculations that follow.

\footnotetext{
${ }^{4}$ The same observation was made in [8].
} 


\subsection{Construction of Weyl tensor local operators}

Now we can construct diffeomorphism-invariants with definite Weyl scaling, which will comprise the basic ingredients of the gauge-invariant operators we will discuss later on. Starting with an arbitrary scalar $Y$, we can construct a four-derivative invariant by taking the norm-squared of the tensor (3.2),

$$
\begin{aligned}
\mathcal{O} \equiv & g^{a c} g^{b d} \hat{\nabla}_{a} \hat{\nabla}_{b} Y \hat{\nabla}_{c} \hat{\nabla}_{d} Y \\
= & \left(\nabla_{a} \nabla_{b} Y\right)\left(\nabla^{a} \nabla^{b} Y\right)+4\left(\partial_{a} \varphi\right)\left(\partial_{b} Y\right)\left(\nabla^{a} \nabla^{b} Y\right)-2\left(\partial_{a} \varphi\right)\left(\partial^{a} Y\right)\left(\nabla^{2} Y\right) \\
& +4(\vec{\partial} \varphi)^{2}(\vec{\partial} Y)^{2}-2\left(\partial_{a} \varphi \partial^{a} Y\right)^{2} .
\end{aligned}
$$

This operator $\mathcal{O}$ is a scalar under diffeomorphisms, and transforms with weight -4 under Weyl transformations, in units where $g_{\bullet \bullet}$ has weight +2 .

Now we consider the case $Y=X^{\mu}$, transforming the expression to unit gauge. We begin by defining

$$
\mathcal{A}_{(a b)(c d)} \equiv \nabla_{a} \nabla_{b} X \cdot \nabla_{c} \nabla_{d} X .
$$

Now we would like to find the unit-gauge expressions for

$$
\hat{\mathcal{A}}_{(a b)(c d)} \equiv \hat{\nabla}_{a} \hat{\nabla}_{b} X \cdot \hat{\nabla}_{c} \hat{\nabla}_{d} X
$$

and

$$
\mathcal{O}_{22} \equiv g^{a c} g^{b d} \hat{\mathcal{A}}_{(a b)(c d)} .
$$

We start by considering just the values for the second derivative, using symmetries, the leading-order EOM, and Virasoro constraints. We obtain

$$
\hat{\nabla}_{b} \hat{\nabla}_{a} X^{\mu}=\hat{\nabla}_{b} \partial_{a} X^{\mu}=\nabla_{b} \partial_{a} X^{\mu}+\partial_{b} \varphi \nabla_{a} X^{\mu}+\partial_{a} \varphi \nabla_{b} X^{\mu}-g_{a b} \partial^{c} \varphi \nabla_{c} X^{\mu},
$$

with the properties

$$
\hat{\nabla}_{b} \hat{\nabla}_{a} X^{\mu}=\hat{\nabla}_{a} \hat{\nabla}_{b} X^{\mu}
$$

and

$$
g^{a b} \hat{\nabla}_{b} \hat{\nabla}_{a} X^{\mu}=g^{a b} \nabla_{b} \nabla_{a} X^{\mu} \simeq 0,
$$

where the $\simeq$ means we are modding out by the leading-order EOM.

In unit gauge, where $g_{a b}=\delta_{a b}$, we have

$$
\hat{\nabla}_{w} \hat{\nabla}_{\bar{w}} X^{\mu}=\hat{\nabla}_{\bar{w}} \hat{\nabla}_{w} X^{\mu} \simeq 0 .
$$

The other components are

$$
\begin{aligned}
& \hat{\nabla}_{w}^{2} X^{\mu}=\partial_{w}^{2} X^{\mu}+2 \partial_{w} \varphi \partial_{w} X^{\mu} \simeq \partial_{w}^{2} X^{\mu}-\frac{\partial_{w}^{2} X \cdot \partial_{\bar{w}} X}{\partial_{w} X \cdot \partial_{\bar{w}} X} \partial_{w} X^{\mu}=\partial_{w}^{2} X^{\mu}-\frac{\mathcal{I}_{21}}{\mathcal{I}_{11}} \partial_{w} X^{\mu}, \\
& \hat{\nabla}_{\bar{w}}^{2} X^{\mu}=\partial_{\bar{w}}^{2} X^{\mu}+2 \partial_{\bar{w}} \varphi \partial_{\bar{w}} X^{\mu} \simeq \partial_{\bar{w}}^{2} X^{\mu}-\frac{\partial_{w} X \cdot \partial_{\bar{w}}^{2} X}{\partial_{w} X \cdot \partial_{\bar{w}} X} \partial_{\bar{w}} X^{\mu}=\partial_{\bar{w}}^{2} X^{\mu}-\frac{\mathcal{I}_{12}}{\mathcal{I}_{11}} \partial_{\bar{w}} X^{\mu},
\end{aligned}
$$

so that

$$
\widehat{\mathcal{I}}_{22} \equiv \hat{\nabla}_{w}^{2} X^{\mu} \hat{\nabla}_{\bar{w}}^{2} X_{\mu}
$$




$$
\begin{aligned}
& =\left(\partial_{w}^{2} X^{\mu}-\frac{\mathcal{I}_{21}}{\mathcal{I}_{11}} \partial_{w} X^{\mu}\right)\left(\partial_{\bar{w}}^{2} X_{\mu}-\frac{\mathcal{I}_{12}}{\mathcal{I}_{11}} \partial_{\bar{w}} X_{\mu}\right) \\
& =\mathcal{I}_{22}-\frac{\mathcal{I}_{12} \mathcal{I}_{21}}{\mathcal{I}_{11}} .
\end{aligned}
$$

Likewise, in Lorentzian signature, we have

$$
\widehat{\mathcal{I}}_{22} \equiv \hat{\nabla}_{+}^{2} X^{\mu} \hat{\nabla}_{-}^{2} X_{\mu}=\mathcal{I}_{22}-\frac{\mathcal{I}_{12} \mathcal{I}_{21}}{\mathcal{I}_{11}}
$$

The expression for this invariant in unit gauge can also be written as

$$
\widehat{\mathcal{I}}_{22}=\mathcal{I}_{11} \partial \bar{\partial}\left[\ln \left(\mathcal{I}_{11}\right)\right] .
$$

We will use the invariant $\widehat{\mathcal{I}}_{22}$ in subsequent discussions to construct higher-dimension operators that will play a role as terms in the action with adjustable coefficients.

The operator $\widehat{\mathcal{I}}_{22}$, and functions of it and $\mathcal{I}_{11}$, are the only Weyl-invariant scalar operators that can be constructed using two or fewer derivatives. There is another candidate operator with only single- and double-derivatives acting on $X^{\mu}$, but we will see now that this operator vanishes. That is,

$$
\mathcal{O}^{(\text {six-derivative })} \equiv\left(\hat{\nabla}_{a} X \cdot \hat{\nabla}_{b} \hat{\nabla}_{c} X\right)\left(\hat{\nabla}^{a} X \cdot \hat{\nabla}^{b} \hat{\nabla}^{c} X\right)
$$

vanishes. This fact is easily understood in conformal gauge in the basis of the non-Weylcovariant $\mathcal{I}_{p q}$. The difference between any covariant $\widehat{\mathcal{I}}_{p q}$ and its non-covariant version $\mathcal{I}_{p q}$ involves a set of correction terms that can be written in terms of $\mathcal{I}_{r s}$, with $r<p$ or $s<q$, or both. The difference between the Weyl-covariant and non-Weyl-covariant versions of $\mathcal{I}_{12} \mathcal{I}_{21}$ would have to contain only $\mathcal{I}_{11}^{3}$. Terms with a single $\mathcal{I}_{12}$ or a single $\mathcal{I}_{21}$, but not both, would have to have spin \pm 1 and could not be a scalar. So the the operator $\mathcal{O}^{\text {(six-derivative) }}$ can only be proportional to a sum of $\mathcal{I}_{12} \mathcal{I}_{21}$ and $\mathcal{I}_{11}^{3}$. Neither of these can appear. The term $\mathcal{I}_{11}^{3}$ cannot appear because the Weyl-covariant derivative preserves the $X$-scaling of an operator. So $\mathcal{I}_{11}^{3}$, which has $X$-scaling +6 , cannot appear in a covariant version of $\mathcal{I}_{12} \mathcal{I}_{21}$, which has $X$-scaling +4 . The noncovariant $\mathcal{I}_{12} \mathcal{I}_{21}$ has the correct weight under rigid rescalings, but does not transform as a tensor under general conformal transformations, as it is proportional to $\mathcal{I}_{11}^{2}\left(\partial \ln \left(\mathcal{I}_{11}\right)\right)\left(\bar{\partial} \ln \left(\mathcal{I}_{11}\right)\right)$. Indeed, the noncovariance of $\mathcal{I}_{12} \mathcal{I}_{21}$ under Weyl transformations is the key property that allows it to appear as the numerator of the classical term cancelling the quantum-mechanical Weyl anomaly.

\subsection{Higher-dimension operators in the string effective action}

\subsubsection{Conformal dimension and $X$-scaling}

In conformal gauge, all operators suitable for addition to the action are of course dimension 2. This is logically necessary because conformal invariance is a residual gauge symmetry of the action. However, effective field theory is still applicable in the sense that operators are arranged hierarchically in terms of their $X$-scaling, with a maximum $X$-scaling appearing $(X$-scaling +2$)$, and with the relevance of operators in the long-string approximation determined by their $X$-scalings. 
Assuming the basic symmetries of the problem - the target-space Poincaré symmetry and the worldsheet gauge symmetries - we can classify all operators up to a given $X$ scaling, modulo total derivatives and terms proportional to the leading-order equations of motion and leading-order Virasoro constraints. For straight, static strings, such as those considered in $[3,7,9-16,23,24]$, the computation of any amplitude to order $\alpha^{\prime p} / R^{2 p}$ relative to its classical value requires including all possible terms in the effective action of order up to and including $|X|^{-2(p-1)}$. For rotating strings, such as those considered in [17-21], computation of amplitudes to order $J^{-p}$, relative to the classical value, requires including the same set of operators.

\subsubsection{The $\mathcal{I}_{11}$-dressing rule}

The problem is simplified by the hypothesis that the only quantity allowed to appear raised to negative powers in local operators, is the invariant $\mathcal{I}_{11}$. Treating this as an exact property of the effective string expansion, order by order in the inverse size of the string, can be justified on several grounds. First, all other Poincaré-invariant combinations $\mathcal{I}_{p q}$ vanish in the static string configuration. Therefore, bilinears $\mathcal{I}_{p q}$ with $p$ or $q$ greater than or equal to 2 cannot appear as denominators in any effective action that can be used to describe a long, approximately static string. Second, the invariant $\mathcal{I}_{11}$ is the operator with the largest ratio of $X$-scaling to worldsheet scaling dimension. Thus, any expression with homogeneous worldsheet scaling dimension that can be expanded as a sum of terms will be dominated at large $X$ by the terms with the most powers of $\mathcal{I}_{11}$. For rational expressions, where both the numerator and denominator are polynomials in multi-derivatives of $X$, the denominator in particular is dominated by terms with the largest number of powers of $\mathcal{I}_{11}$. Thus the rational function in question can be expanded at large $X$, where the denominator will always be dominated by the term with the largest number of powers of $\mathcal{I}_{11}$.

For instance, the term $\mathcal{O} \equiv \frac{{\widehat{\mathcal{I}_{33}}}^{2}}{{\mathcal{\mathcal { I } _ { 1 1 } ^ { 5 }}}_{\mathcal{I}_{22}}^{2} \mathcal{I}_{11}}$ can be expanded at large $X$ to give

$$
\mathcal{O}=\frac{{\widehat{\mathcal{I}_{33}}}^{2}}{\mathcal{I}_{11}^{5}}-\frac{{\widehat{\mathcal{I}_{33}}}^{2}{\widehat{\mathcal{I}_{22}}}^{2}}{\mathcal{I}_{11}^{9}}+\cdots
$$

with the omitted terms being of order $\widehat{\mathcal{I}}_{33}^{2}{\widehat{\mathcal{I}_{22}}}^{4} / \mathcal{I}_{11}^{13}=O\left(|X|^{-14}\right)$. Terms such as $\widehat{\mathcal{I}}_{33}^{2} / \widehat{\mathcal{I}}^{22}{ }^{2}$ are consistent with all the necessary symmetries, but they are singular in the long string vacuum and thus describe a different universality class from the conventional effective string theory. They are also fine-tuned, in the sense that a small perturbation of the microscopic theory could be expected to change the denominator from ${\widehat{\mathcal{I}_{22}}}^{2}$ to ${\widehat{\mathcal{I}_{22}}}^{2}+\epsilon^{\prime} \mathcal{I}_{11} \mathcal{I}_{33}+\epsilon \mathcal{I}_{11}^{4}$ for some small $\epsilon$ and $\epsilon^{\prime}$. These three terms have $X$-scaling $|X|^{4},|X|^{4}$, and $|X|^{8}$, respectively, so no matter how small the value of $\epsilon$, the denominator will always be dominated by the $\mathcal{I}_{11}^{4}$ term for string solutions with a sufficiently long scale $|X| \sim R$. For the theory to be dominated by ${\widehat{\mathcal{I}_{22}}}^{2}$ in the long-string limit, the coefficients $\epsilon, \epsilon^{\prime}$ would have to be fine-tuned exactly to zero, in the absence of any symmetry principle that would enforce this independently. The $\mathcal{I}_{11}$ dressing rule is, in this sense, just a corollary of the principle of naturalness.

The third and most concrete justification for the $\mathcal{I}_{11}$-dressing rule is that it appears to be true. We know of several examples of microscopically well-defined holographic theories 
that give rise to effective string theories in $4 \mathrm{D}$, after integrating out the motions of the string into the fifth (and higher) dimension. In each of these examples, the dressing rule appears to hold. For instance, the effective strings generated by holographic compactifications [3] obey the rule, as does the effective theory generated by the irrelevantly-perturbed Liouville theory discussed at the end of [1], and the effective theory in an early derivation the $4 \mathrm{D}$ effective string from warped compactification with a minimum of the warp factor [4]. Though the analysis in these works was at the classical and one-loop level, it is clear that the dressing rule holds in these models to all loops. The mass $M_{\text {holo }}$ of the fluctuations into the holographic direction (the mass being taken with respect to conformal time on the worldsheet) is proportional to $\mathcal{I}_{11}$ at large $|X|$, so when one integrates out the holographic direction the singular operators derive from inverse powers of $M_{\text {holo }}$, and thus have $\mathcal{I}_{11}$ dressing.

The dressing rule must clearly break down near a point on the worldsheet where $\mathcal{I}_{11}$ vanishes. Such points appear on the worldsheets of any realistic rotating string solution in four dimensions, such as closed strings or open strings with Neumann boundary conditions. In the vicinity of such points the $\mathcal{I}_{11}$-dressing rule is violated, but the effective theory need not itself break down or include additional light degrees of freedom. In the case of strings with massless quarks, the singularity is resolved by a reorganization of operators dressed with $\mathcal{I}_{22}$, rather than $\mathcal{I}_{11}$ [21]. This reorganization of operators is physically interesting and may be testable experimentally: the emergence of the $\mathcal{I}_{22}$ dressing is associated with fractional powers of angular momentum appearing in the formula for the large- $J$ meson spectrum [18-20, 25]. We will not deal with such situations in this paper, however; we restrict our attention to local properties of the worldsheet effective theory away from loci where $\mathcal{I}_{11}$ vanishes.

\subsubsection{Scalar operators of conformal dimension 2}

Assuming the validity of the $\mathcal{I}_{11}$-dressing rule, the enumeration of all gauge-invariant operators at a given order in $|X|$ becomes a finite problem. The set of Poincaré invariant terms is generated by the bilinears $\mathcal{I}_{p q}$, together with $\mathcal{H}_{(p q)} \equiv \partial^{p} X \cdot \partial^{q} X$ and $\tilde{\mathcal{H}}_{(p q)} \equiv \bar{\partial}^{p} X \cdot \bar{\partial}^{q} X$. These operators, and their covariantized versions $\widehat{\mathcal{I}_{p q}}, \widehat{\mathcal{H}_{(p q)}}, \widehat{\tilde{\mathcal{H}}_{(p q)}}$, have scaling dimension $p+q$. To classify possible terms in the action, we can write each operator uniquely as an undressed numerator (i.e., a monomial or polynomial in $\mathcal{H}_{(r s)}, \tilde{\mathcal{H}}_{(t u)}$, and $\mathcal{I}_{p q}$ with $p$ or $q \geq 2$ ) dressed with a power of $\mathcal{I}_{11}$. We may then discard all terms proportional to leading-order Virasoro constraints and total derivatives. ${ }^{5}$

The power of $\mathcal{I}_{11}$ dressing a given undressed operator is dictated uniquely by scale invariance, with an underssed scalar operator

$$
\mathcal{O}^{\text {(undressed })} \equiv \prod_{i} \mathcal{I}_{p_{i} q_{i}} \prod_{j} \mathcal{H}_{\left(r_{j} s_{j}\right)} \prod_{k} \tilde{\mathcal{H}}_{\left(t_{k} u_{k}\right)}
$$

dressed to marginality as

$$
\mathcal{O}^{\text {(dressed) }} \equiv \mathcal{I}_{11}^{-\Delta} \mathcal{O}^{\text {(undressed) }},
$$

\footnotetext{
${ }^{5}$ We have chosen our basis of operators so that terms proportional to the leading-order EOM $\partial \bar{\partial} X$ are already omitted.
} 


$$
\Delta=-1+\sum_{i} p_{i}+\sum_{j}\left[r_{j}+s_{j}\right]=-1+\sum_{i} q_{i}+\sum_{k}\left[t_{k}+u_{k}\right] .
$$

The equality of the latter two expressions is simply the constraint that the operator be a scalar field. The $X$-scaling of the undressed operator is $2\left(N_{\mathcal{I}}+N_{\mathcal{H}}+N_{\tilde{\mathcal{H}}}\right)=\sum_{i} 2+\sum_{j} 2+$ $\sum_{k} 2$, so the $X$-scaling of the dressed operator is

$$
\begin{aligned}
\operatorname{Scale}_{\mathrm{X}}\left[\mathcal{O}^{(\text {dressed })}\right] & =-2 \Delta+\operatorname{Scale}_{\mathrm{X}}\left[\mathcal{O}^{\text {(undressed })}\right] \\
& =2-\sum_{i} 2\left(p_{i}-1\right)-\sum_{j}\left[2\left(r_{j}-1\right)+2\left(s_{j}-1\right)\right] \\
& =2-\sum_{i} 2\left(q_{i}-1\right)-\sum_{k}\left[2\left(t_{k}-1\right)+2\left(u_{k}-1\right)\right] .
\end{aligned}
$$

We can write this symmetrically as

$$
\begin{aligned}
\operatorname{Scale}_{\mathrm{X}}\left[\mathcal{O}^{(\text {dressed })}\right]= & 2-\sum_{i}\left[\left(p_{i}-1\right)+\left(q_{i}-1\right)\right] \\
& -\sum_{j}\left[\left(r_{i}-1\right)+\left(s_{i}-1\right)\right]-\sum_{k}\left[\left(t_{k}-1\right)+\left(u_{k}-1\right)\right] .
\end{aligned}
$$

The invariants $\mathcal{H}_{(11)}$ and $\tilde{\mathcal{H}}_{(11)}$ are proportional to the leading-order Virasoro constraints, so every $\mathcal{I}, \mathcal{H}$ or $\tilde{\mathcal{H}}$ added to an operator contributes negatively to the $X$-scaling, from the starting point of the tree-level action $\mathcal{I}_{11}$, with $X$-scaling 2 .

\subsection{Universality and nonuniversality}

In this section we will analyze the issue of universality of the action at the first two subleading orders in the $1 / R$ expansion, where $R$ is the typical length scale of the string. We will emphasize that all amplitudes are universal at next-to-leading order (NLO). At nextto-next-to-leading order (NNLO), it will emerge that certain observables are universal and some are not, and we will explain why this is so, giving a useful criterion to distinguish between the two cases.

\subsubsection{Universality at NLO}

To classify possible marginal operators with $X$-scaling 0 , we must, according to (3.8), find all undressed operators with

$$
\sum_{i}\left[\left(p_{i}-1\right)+\left(q_{i}-1\right)\right]+\sum_{j}\left[\left(r_{i}-1\right)+\left(s_{i}-1\right)\right]+\sum_{k}\left[\left(t_{k}-1\right)+\left(u_{k}-1\right)\right]=2 .
$$

The only such operators are $\mathcal{H}_{(12)} \tilde{\mathcal{H}}_{(12)}, \mathcal{I}_{12} \mathcal{I}_{21}$ and $\widehat{\mathcal{I}_{22}}$. The first vanishes, modulo operators with lower $X$-scaling, by the Virasoro constraints. The second, as discussed above, does not transform covariantly under Weyl transformations, cannot be rendered covariant with additional terms, and does not correspond to a gauge-invariant operator after dressing with two negative powers of $\mathcal{I}_{11}$. This leaves us with the Weyl-covariant scalar $\widehat{\mathcal{I}_{22}}$, which is dressed to marginality as $\widehat{\mathcal{I}_{22}} / \mathcal{I}_{11}$. From the form (3.6), we see this operator is proportional to a total derivative, and does not affect the string dynamics except possibly as a 
boundary term. This leaves no adjustable operators in the string action at next-to-leading order in the large $X$ expansion for closed strings. This argument justifies the claim of universality of the asymptotic Regge intercept for closed strings with angular momentum in two planes in $D \geq 5 .^{6}$

\subsubsection{Nonuniversality at NNLO}

A similar analysis can be applied to classify operators at NNLO. Here, we need to find undressed operators with

$$
\sum_{i}\left[\left(p_{i}-1\right)+\left(q_{i}-1\right)\right]+\sum_{j}\left[\left(r_{i}-1\right)+\left(s_{i}-1\right)\right]+\sum_{k}\left[\left(t_{k}-1\right)+\left(u_{k}-1\right)\right]=4 .
$$

There are many such operators, for instance:

$$
\begin{aligned}
& \mathcal{O}_{1}^{\text {(undressed) }} \equiv\left(\widehat{\mathcal{I}_{22}}\right)^{2}, \\
& \mathcal{O}_{2}^{\text {(undressed) }} \equiv \widehat{\widehat{\mathcal{I}}_{22}} \mathcal{I}_{12} \mathcal{I}_{21}, \quad \mathcal{O}_{3}^{\text {(undressed) }} \equiv\left(\mathcal{I}_{12} \mathcal{I}_{21}\right)^{2} \text {, } \\
& \mathcal{O}_{4}^{\text {(undressed) }} \equiv \widehat{\mathcal{I}}_{22} \mathcal{H}_{(12)} \tilde{\mathcal{H}}_{(12)}, \quad \quad \mathcal{O}_{5}^{\text {(undressed) }} \equiv \mathcal{H}_{(12)}^{2} \tilde{\mathcal{H}}_{(12)}^{2} \text {. }
\end{aligned}
$$

Most of these can be eliminated as candidate terms in the action. Operators $\mathcal{O}_{2}^{\text {(undressed) }}$ and $\mathcal{O}_{3}^{\text {(undressed) }}$ contain the expression $\mathcal{I}_{12} \mathcal{I}_{21}$, which is noncovariant, and cannot be rendered covariant, and no linear combination of $\mathcal{O}_{2}^{\text {(undressed) }}$ and $\mathcal{O}_{3}^{\text {(undressed) }}$ is covariant either. The operators $\mathcal{O}_{4}^{\text {(undressed) }}$ and $\mathcal{O}_{5}^{\text {(undressed) }}$ are proportional to the first derivatives of free stress tensors, and so vanish by the Virasoro constraints, modulo operators with smaller $X$-scaling. The operator $\mathcal{O}_{1}^{\text {(dressed) }} \equiv\left(\widehat{\mathcal{I}_{22}}\right)^{2} / \mathcal{I}_{11}^{3}$ is nonzero and independent as a possible term in the effective Lagrangian, however. In gauge-invariant language, this corresponds to the curvature-squared of the induced metric. As a result, the predictions of effective string theory are nonuniversal at NNLO in the long string expansion.

The end of universality does not mean the end of the usefulness of effective string theory, however. The small number of adjustable terms means that there are far more observables than parameters at NNLO, so one can derive long-string or large- $J$ sum rules that are universal at NNLO, because the adjustable coefficients cancel out in certain combinations of observables. Also, in many situations, such as that of a static string, the term $\mathcal{O}_{1}^{\text {(dressed) }}$ does not contribute at NNLO because its classical value vanishes. Therefore, it can only contribute through quantum fluctuations, which are suppressed by additional powers of $\alpha^{\prime} / R^{2}$. As a result, the energy spectrum of the static string is universal up to and including order $\alpha^{\prime} R^{-3}$, rather than just $R^{-1}$. This NNLO universality holds only for the static string and other cases where $\mathcal{O}_{1}^{\text {(dressed) }}$ vanishes classically. It does not apply, for instance, to the case of the rotating string, so we should expect the meson mass spectrum to be sensitive to the coefficient of the curvature-squared term at order $J^{-\frac{3}{2}}$.

\footnotetext{
${ }^{6}$ These arguments do not apply without modification to the case of strings with folds or Neumann boundaries, in which cases the dressing hypothesis breaks down. Note, however, that in [21] a similar analysis was applied to derive a universal asymptotic intercept for open string Regge trajectories under the hypothesis of an $\mathcal{I}_{22}$ dressing rule for Neumann boundaries.
} 


\section{Interaction corrections to the OPE}

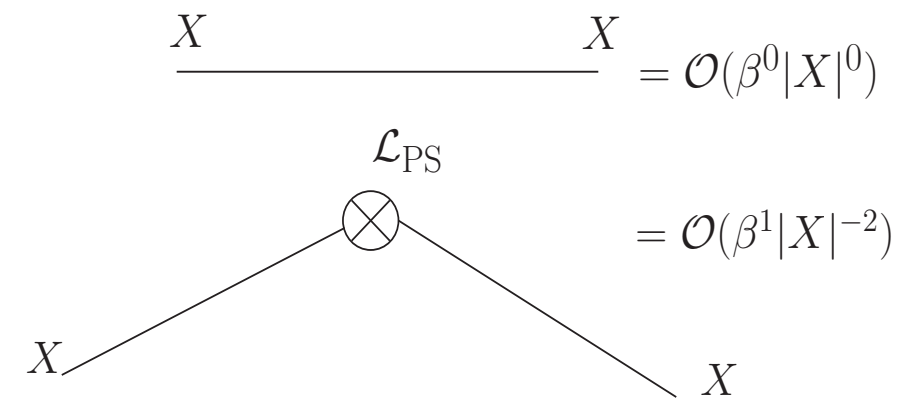

\subsection{The propagator correction}

In the presence of the interaction term, the theory preserves conformal invariance but is no longer a free conformal field theory. The structure of the operator product expansion becomes less simple when $X$ is no longer a free field. The propagator for the fluctuation $Y^{\mu}=X^{\mu}-E^{\mu}$ of the embedding coordinates receives non-vanishing corrections at relative order $\beta|X|^{-2}$, and these corrections affect the operator product expansions of local operators with one another.

The form of the leading-order propagator correction is generally not simple. The correction satisfies a differential equation controlled by the $\beta^{1}|Y|^{1}$ terms in the expansion of $X$ in the EOM (2.15) as the solution $X^{\mu}=E^{\mu}+Y^{\mu}$. The full set of terms in the EOM for the propagator correction is lengthy, and we do not reproduce it here. Rather, we will deal directly with the effect of the propagator correction on the OPE of the stress tensor with other operators.

Note that the propagator correction vanishes at first subleading order in the case of the static string $[3,7,9-16,23,24]$. The corrections to the tree-level propagator are all proportional to

$$
\left.E_{p q} \equiv \mathcal{I}_{p q}\right|_{X=E}=\partial^{p} E \cdot \bar{\partial}^{q} E,
$$

with $p$ and/or $q$ greater than unity, and all such expectation values of higher-derivative invariants vanish in the classical solution for the static case. This is not so for more generic situations, such as the rotating string. The propagator is used to compute vertex operator correlation functions, as well as to verify the consistency of the gauge-fixed theory via the stress tensor OPE. We therefore need to understand the propagator correction to some extent.

\subsection{Nonrenormalization of the OPE between $T$ and $X$ at $O\left(\beta^{1}\right)$}

We will see that the propagator correction, despite modifying the operator product expansion of the $X$ field, does not affect its conformal properties. This nontrivial fact, which is not manifest in the old covariant formalism of [1], can be traced to our embedding of the effective string in the Polyakov path integral. 
In this subsection, the OPE of $T$ with $X$ is examined. Although there are the propagator corrections and the contribution from $T^{\left[\beta^{1}\right]}$, it will turn out that

$$
T\left(w_{1}\right) X^{\mu}\left(w_{2}\right)=\frac{\partial X^{\mu}\left(w_{2}\right)}{w_{12}}+(\text { smooth terms })+O\left(|X|^{-3}\right) .
$$

To prove this, we first investigate the OPE of $T^{\left[\beta^{0}\right]}$ with $X$, where we will see that

$$
\left.T^{\left[\beta^{0}\right]}\left(w_{1}\right) X^{\mu}\left(w_{2}\right)\right|_{\text {prop. correction }}=-T^{\left[\beta^{1}\right]}\left(w_{1}\right) X^{\mu}\left(w_{2}\right)+(\text { smooth terms })+O\left(|X|^{-3}\right) .
$$

The contraction of $T^{\left[\beta^{0}\right]}$ with the PS vertex $-S^{\left[\beta^{1}\right]}=-\int d^{2} w \mathcal{L}_{\mathrm{PS}}$ is

$$
\begin{aligned}
T^{[0]}\left(w_{1}\right) \cdot\left(-S^{[\beta]}\right)= & 2 \beta \partial X_{\mu} \partial X^{\mu} \frac{\partial \bar{\partial} \varphi}{\mathcal{I}_{11}}\left(w_{1}\right) \\
& -\frac{\beta}{\pi} \int d^{2} w_{3} \frac{\partial X_{\mu}\left(w_{1}\right) \bar{\partial} X^{\mu}\left(w_{3}\right)}{w_{13}^{2}} \frac{\partial \bar{\partial} \varphi}{\mathcal{I}_{11}}\left(w_{3}\right)+\mathcal{O}\left(|X|^{-2}\right) .
\end{aligned}
$$

By partial integration, and using the leading order EOM, the second term in the r.h.s. becomes

$$
\begin{aligned}
\int d^{2} w_{3} & \frac{\partial X_{\mu}\left(w_{1}\right) \bar{\partial} X^{\mu}\left(w_{3}\right)}{w_{13}^{2}} \frac{\partial \bar{\partial} \varphi}{\mathcal{I}_{11}}\left(w_{3}\right) \\
= & \int d^{2} w_{3}\left[\partial^{2} \varphi\left(w_{3}\right)+\left(\partial \varphi\left(w_{3}\right)\right)^{2}\right] \bar{\partial}_{3}\left[\frac{1}{w_{13}} \frac{\partial X_{\mu}\left(w_{1}\right) \bar{\partial} X^{\mu}\left(w_{3}\right)}{\mathcal{I}_{11}\left(w_{3}\right)}\right] \\
= & -2 \pi\left[\partial^{2} \varphi\left(w_{1}\right)+\left(\partial \varphi\left(w_{1}\right)\right)^{2}\right] \\
& +\int d^{2} w_{3}\left[\partial^{2} \varphi\left(w_{3}\right)+\left(\partial \varphi\left(w_{3}\right)\right)^{2}\right] \sum_{n=1}^{\infty} \frac{w_{13}^{n-1}}{n !} \bar{\partial} \frac{\partial^{n} \mathcal{I}_{11}}{\mathcal{I}_{11}}\left(w_{3}\right)
\end{aligned}
$$

The latter term can be neglected because when contracting with $X^{\mu}\left(w_{2}\right)$, it does not yield any singularity in the limit $w_{1}-w_{2} \rightarrow 0$. Finally,

$$
\begin{aligned}
T^{[0]}\left(w_{1}\right) \cdot\left(-S^{[\beta]}\right)= & T^{[\beta]}\left(w_{1}\right) \\
& +(\text { terms which are negligible in the above sense }) \\
& +\mathcal{O}\left(X^{-2}\right) .
\end{aligned}
$$

Contracting with $X^{\mu}\left(w_{2}\right)$, we get

$$
\left.T^{[0]}\left(w_{1}\right) X^{\mu}\left(w_{2}\right)\right|_{\text {prop. correction }}=-T^{[\beta]}\left(w_{1}\right) X^{\mu}\left(w_{2}\right)+(\text { smooth })+O\left(|X|^{-3}\right) .
$$

The result is that $T^{\left[\beta^{0}+\beta^{1}\right]}\left(w_{1}\right) X^{\mu}\left(w_{2}\right)=\frac{1}{w_{12}} \partial X^{\mu}\left(w_{2}\right)+($ smooth $)+O\left(|X|^{-3}\right)$, where the contractions are performed with the full propagator. To put it more concisely,

$$
T^{[\mathrm{first} \text { two orders in } \beta]}\left(w_{1}\right) X^{\mu}\left(w_{2}\right)=\frac{1}{w_{12}} \partial X^{\mu}\left(w_{2}\right)+(\text { smooth })+O\left(|X|^{-3}\right),
$$

where the OPE is performed in the full quantum theory. In other words, the OPE of $T^{[\mathrm{fulll}]}$ with $X$ in the full interacting theory is the same as that of the free $T^{\left[\beta^{0}\right]}$ in the free theory, modulo terms of order $|X|^{-3}$ and smaller. 
This proves the standard OPE of $T$ with $X$ is unmodified by interaction and quantum corrections up to and including relative order $\beta|X|^{-2}$. In other words, to first subleading order, the conformal transformation of $X$ remains that of a primary field of weight zero. This was inevitable, as we formulated our theory from the outset with explicit Weyl invariance built in, from which the conformal invariance of the gauge-fixed theory is inherited. The operators in the CFT describing the theory in conformal gauge receive their conformal properties from the Weyl transformations of the fields in the path integral. Since our construction of the Weyl-invariant quantum theory was based on assigning $X$ to be invariant under Weyl transformations, it automatically appears as a conformal primary of weight zero in the interacting CFT.

\subsection{Closure of the OPE of $T$ with itself}

We now demonstrate that the OPE of $T$ with itself satisfies the standard form, with $c=D+12 \beta=26$, up to and including $\mathcal{O}\left(|X|^{-1}\right)$, using the result of the previous section. First, when we separate $X^{\mu}=E^{\mu}+Y^{\mu}$, where $E^{\mu}$ is a $c$-number solution of $\partial \bar{\partial} E^{\mu}=0$ and $Y^{\mu}$ is an operator representing fluctuations, the $T X$ OPE is the same as the TY OPE:

$$
T^{\left[\beta^{0}+\beta^{1}\right]}\left(w_{1}\right) Y^{\mu}\left(w_{2}\right) \sim \frac{1}{w_{12}} \partial\left(E^{\mu}+Y^{\mu}\right)\left(w_{2}\right)+\mathcal{O}\left(E^{-3}\right) .
$$

Then, expanding $T$ with respect to $Y$,

$$
\begin{aligned}
T^{\left[\beta^{0}+\beta^{1}\right]}=\left.T^{\left[\beta^{0}+\beta^{1}\right]}\right|_{X=E}-\frac{1}{\alpha^{\prime}}(2 \partial E \cdot \partial Y+\partial Y \cdot \partial Y) & \\
+\beta & {\left[-\partial E \cdot \partial E\left(\frac{\partial E \cdot \bar{\partial} Y+\partial Y \cdot \bar{\partial} E}{E_{11}^{2}} \partial \bar{\partial} \log E_{11}-\frac{1}{E_{11}} \partial \bar{\partial} \frac{\partial E \cdot \bar{\partial} Y+\partial Y \cdot \bar{\partial} E}{E_{11}}\right)\right.} \\
& \left.\quad+2 \partial E \cdot \partial Y \frac{\partial \bar{\partial} \ln E_{11}}{E_{11}}+\partial^{2} \frac{\partial E \cdot \bar{\partial} Y+\partial Y \cdot \bar{\partial} E}{E_{11}^{2}}-\partial \ln E_{11} \partial \frac{\partial E \cdot \bar{\partial} Y+\partial Y \cdot \bar{\partial} E}{E_{11}^{2}}\right] \\
& +\mathcal{O}\left(Y^{2} E^{-2}\right)
\end{aligned}
$$

with $E_{11}$ as in (4.1). A tedious but straightforward calculation using eq. (4.5) gives

$$
\begin{aligned}
T^{\left[\beta^{0}+\beta^{1}\right]}\left(w_{1}\right) T^{\left[\beta^{0}+\beta^{1}\right]}\left(w_{2}\right) \sim & \frac{D+12 \beta}{2 w_{12}^{4}}+\frac{2}{w_{12}^{2}} T^{\left[\beta^{0}+\beta^{1}\right]}\left(w_{2}\right) \\
& +\frac{1}{w_{12}} \partial T^{\left[\beta^{0}+\beta^{1}\right]}\left(w_{2}\right)+\mathcal{O}\left(|X|^{-2}\right)
\end{aligned}
$$

This OPE implies that the theory is a conformal field theory with $c=26$, regardless of the space-time dimensions and the form of the classical solution. This result generalizes the stress tensor OPE in [1] in the special case where $E^{\mu}$ is linear in the worldsheet coordinates.

\subsection{OPE of $T$ with composite operators}

Suppose $\mathcal{O}$ is some operator with definite or polynomial $|X|$-scaling $|X|^{p}$, and we would like to compute the OPE of $T$ with $\mathcal{O}$ up to (and excluding) terms of order $|X|^{-4}$, relative to $\mathcal{O}$ itself. $^{7}$

\footnotetext{
${ }^{7}$ That is, we would like to compute the OPE of $T$ with $\mathcal{O}$ up to (and excluding) terms of order $|X|^{p-4}$.
} 
Terms involving cubic or higher interaction vertices are too small to contribute. To connect $T$ and $\mathcal{O}$ with a cubic interaction vertex, we would need to pull three $X$ fields from $T$ and $\mathcal{O}$, and the cubic interaction vertex from the PS term itself scales as $|X|^{-3}$. Thus, the total effect scales as $|X|^{-6}$, relative to the classical combined $X$-scaling of $T \mathcal{O}$, the latter being $|X|^{p+2}$. So any contribution to the $T \mathcal{O}$ OPE involving a cubic vertex is no larger than $|X|^{p-4}$. Likewise, a diagram with a $q^{\text {th }}$-order vertex, with $q \geq 4$, must connect the two operators by pulling off at least one $X$ field from each, and the vertex itself scales as $|X|^{-q}$, so the $|X|$-scaling of the contribution is $|X|^{-q-2}$, relative to the classical $|X|$ scaling $|X|^{p+2}$ of the classical operator product $T \mathcal{O}$. The $|X|$-scaling of the term is thus $|X|^{p-q}$ or smaller, and thus no contribution with a quartic or higher interaction vertex will contribute to the OPE at the desired order of accuracy.

Therefore, the only terms contributing to the TO OPE up to the order of interest are classical propagators, with or without interaction corrections. For a single propagator, we have found that the effect of the propagator correction on the singular terms in the OPE precisely cancels the effect of the explicit order- $\beta^{1}|X|^{0}$ correction to the stress tensor. For two propagators, a double contraction with leading-order propagators is order- $|X|^{-4}$, relative to the classical operator product, and each interaction correction to a propagator suppresses the $|X|$-scaling further by $|X|^{-2}$. A single interaction correction to either propagator is already order- $|X|^{p-4}$ in total, and thus small enough to ignore. Likewise, contractions using three or more propagators, even uncorrected propagators, is already order- $|X|^{p-4}$, and can be ignored as well.

Thus, the OPE of $T^{[\mathrm{full}]}$ with $\mathcal{O}$ is given by a sum of the following:

- Terms coming from contractions with a single (corrected) propagator, which implement a classical conformal transformation with $X$ transforming as a scalar that is invariant under Weyl transformations;

- An anomalous term coming from a double contraction using free propagators;

- Terms of $X$-scaling $|X|^{p-4}$ or smaller;

- Smooth terms.

For purposes of classifying conformal primary operators at next-to-leading order in the effective string expansion, we care about the first two contributions only.

\section{Conclusions}

We have embedded effective string theory in the Polyakov formalism, so that the standard worldsheet diffeomorphism and Weyl symmetries are manifest. The absence of the Weyl anomaly is clearly proven prior to gauge fixing. The classification of diffeomorphisminvariant operators with definite Weyl scaling implies that no adjustable parameter exists at next-to-leading order, whereas at next-to-next-to-leading order the action is not universal.

One of the efficiencies of our formalism, relative to the old covariant one, is that the primary conformal transformations of $X$ are maintained in the presence of the interaction, 
by construction. Thus, to classify conformal primaries at next-to-leading order, only a classical conformal transformation and a double contraction using free propagators need be taken into account.

\section{Acknowledgments}

$\mathrm{SH}$ is grateful to the Weizmann institute for hospitality while this work was in progress, and would also like to thank J. Sonnenschein, O. Aharony, and M. Field for extremely useful discussions during his visit. JM thanks the Stanford Institute for Theoretical Physics for hospitality during the completion of this paper. The work of SH, SM, and JM is supported by the World Premier International Research Center Initiative (WPI Initiative), MEXT, Japan. The work of SH was also supported in part by Grant-in-Aid for Scientific Research (22740153) and Grant-in-Aid for Scientific Research (26400242) from the Japan Society for Promotion of Science (JSPS).

Open Access. This article is distributed under the terms of the Creative Commons Attribution License (CC-BY 4.0), which permits any use, distribution and reproduction in any medium, provided the original author(s) and source are credited.

\section{References}

[1] J. Polchinski and A. Strominger, Effective string theory, Phys. Rev. Lett. 67 (1991) 1681 [INSPIRE].

[2] O. Aharony, S.S. Gubser, J.M. Maldacena, H. Ooguri and Y. Oz, Large-N field theories, string theory and gravity, Phys. Rept. 323 (2000) 183 [hep-th/9905111] [INSPIRE].

[3] O. Aharony and Z. Komargodski, The effective theory of long strings, JHEP 05 (2013) 118 [arXiv: 1302.6257] [INSPIRE].

[4] M. Natsuume, Nonlinear $\sigma$-model for string solitons, Phys. Rev. D 48 (1993) 835 [hep-th/9206062] [INSPIRE].

[5] M. Lüscher, K. Symanzik and P. Weisz, Anomalies of the free loop wave equation in the WKB approximation, Nucl. Phys. B 173 (1980) 365 [INSPIRE].

[6] M. Lüscher, Symmetry breaking aspects of the roughening transition in gauge theories, Nucl. Phys. B 180 (1981) 317 [INSPIRE].

[7] M. Lüscher and P. Weisz, String excitation energies in $\mathrm{SU}(N)$ gauge theories beyond the free-string approximation, JHEP 07 (2004) 014 [hep-th/0406205] [INSPIRE].

[8] N.D. Hari Dass and P. Matlock, Covariant calculus for effective string theories, Indian J. Phys. 88 (2014) 965 [arXiv:0709.1765] [INSPIRE].

[9] S. Dubovsky, R. Flauger and V. Gorbenko, Effective string theory revisited, JHEP 09 (2012) 044 [arXiv: 1203.1054] [INSPIRE].

[10] M. Lüscher and P. Weisz, Quark confinement and the bosonic string, JHEP 07 (2002) 049 [hep-lat/0207003] [INSPIRE].

[11] O. Aharony and N. Klinghoffer, Corrections to Nambu-Goto energy levels from the effective string action, JHEP 12 (2010) 058 [arXiv: 1008.2648] [INSPIRE]. 
[12] O. Aharony, M. Field and N. Klinghoffer, The effective string spectrum in the orthogonal gauge, JHEP 04 (2012) 048 [arXiv:1111.5757] [INSPIRE].

[13] O. Aharony and E. Karzbrun, On the effective action of confining strings, JHEP 06 (2009) 012 [arXiv: 0903.1927] [INSPIRE].

[14] O. Aharony and M. Field, On the effective theory of long open strings, JHEP 01 (2011) 065 [arXiv: 1008.2636] [INSPIRE].

[15] O. Aharony and M. Dodelson, Effective string theory and nonlinear Lorentz invariance, JHEP 02 (2012) 008 [arXiv: 1111.5758] [INSPIRE].

[16] O. Aharony, Z. Komargodski and A. Schwimmer, The effective action on the confining string, presented by O. Aharony at the Strings 2009 conference, http://strings2009.roma2.infn.it/talks/Aharony_Strings09.ppt, June 2009.

[17] M. Baker and R. Steinke, Effective string theory of vortices and Regge trajectories, Phys. Rev. D 63 (2001) 094013 [hep-ph/0006069] [INSPIRE].

[18] M. Baker and R. Steinke, Semiclassical quantization of effective string theory and Regge trajectories, Phys. Rev. D 65 (2002) 094042 [hep-th/0201169] [INSPIRE].

[19] M. Kruczenski, L.A. Pando Zayas, J. Sonnenschein and D. Vaman, Regge trajectories for mesons in the holographic dual of large- $N_{c}$ QCD, JHEP 06 (2005) 046 [hep-th/0410035] [INSPIRE].

[20] J. Sonnenschein and D. Weissman, Rotating strings confronting PDG mesons, JHEP 08 (2014) 013 [arXiv: 1402.5603] [INSPIRE].

[21] S. Hellerman and I. Swanson, String theory of the Regge intercept, arXiv:1312.0999 [INSPIRE].

[22] J. Polchinski, String theory. Vol. 1: an introduction to the bosonic string, Cambridge Univ. Pr., Cambridge U.K. (1998) [InSPIRE].

[23] J.M. Drummond, Universal subleading spectrum of effective string theory, hep-th/0411017 [INSPIRE].

[24] S. Dubovsky, R. Flauger and V. Gorbenko, Evidence from lattice data for a new particle on the worldsheet of the QCD flux tube, Phys. Rev. Lett. 111 (2013) 062006 [arXiv:1301.2325] [INSPIRE].

[25] F. Wilczek, Diquarks as inspiration and as objects, in From fields to strings, vol. 1, M. Shifman et al. eds., World Scientific, Singapore (2005), pg. 77 [hep-ph/0409168] [INSPIRE]. 